\title{
Parents' Oral Health Literacy and its Impact on their Children's Dental Health Status
}

\author{
Effat Khodadadi ${ }^{1}$, Ayshe Niknahad ${ }^{2}$, Mohammad Mehdi Naghibi Sistani ${ }^{3}$, Mina Motallebnejad ${ }^{4}$
}

\author{
${ }^{1}$ M.Sc. of Pediatric Dentistry, Assistant Professor, Oral Health Research Center, Department of Pedodontics, \\ Faculty of Dentistry, Babol University of Medical Sciences, Babol, Iran \\ ${ }^{2}$ Student Research Committee, Babol University of Medical Sciences, Babol, Iran \\ ${ }^{3}$ Ph.D. of Community Oral Health, Assistant Professor, Oral Health Research Center, Department of Community \\ Oral Health, Faculty of Dentistry, Babol University of Medical Sciences, Babol, Iran \\ ${ }^{4}$ M.Sc. of Oral Medicine, Professor, Oral Health Research Center, Department of Oral Medicine, Faculty of \\ Dentistry, Babol University of Medical Sciences, Babol, Iran
}

\section{Type of article: Original}

\begin{abstract}
Introduction: Because parents play a key role in children's dental health, the aim of this study was to determine the relationship between parents' oral health literacy (OHL) and their children's dental health status in Babol, Iran.

Methods: In this cross sectional study a total of 384 children aged 21 months to 84 months who attended the dental clinic of Babol University of Medical Sciences between September 2015 and February 2016 were examined. We measured dmft index only for primary dentition; during examination the accompanying parent completed the "Oral Health Literacy-Adults Questionnaire". Comparing mean analysis, such as one-way analysis of variance (ANOVA), and an independent-samples t-test, served to compare children's dental caries, missing, and dental fillings' mean differences, between subgroups. In addition, the relationship between OHL, children's dental caries, and dental fillings was assessed using multiple linear regression models while controlling for socioeconomic and demographic factors. All data were analyzed by SPSS version 22.

Results: Children's mean age was 55.1 months (SD: 13.7), while 47\% were girls. Mean children's dental caries, missing, filling, and mean $\mathrm{dmft}$ index were $6.5,0.4,1.2$, and 8.2 respectively. Parents with inadequate OHL had children with more dental caries $(\mathrm{p}=0.005)$, however this relation had no significance while controlling for background factors. Increasing children's dental fillings was significantly related with families living in urban regions ( $\mathrm{p}=0.01,95 \%$ CI: 0.11 to 1.12 ), and parents with adequate OHL ( $\mathrm{p}=0.02,95 \%$ CI: 0.08 to 1.05 ).

Conclusion: Inadequate parents' OHL was associated with children having high dental caries and less dental fillings. Therefore, providing interventions to improve parents' OHL would be valuable in children's dental health promotion programs, especially in countries with a developing oral health system.
\end{abstract}

Keywords: Caregiver, Oral health literacy, Oral health status, Child

\section{Introduction}

The infectious nature of dental caries, its early onset and its early treatment potential requires emphasis on early preventive dental care in children (1). Direct relationship between parents' oral hygiene behavior and their children's oral health-related habits, emphasize the key role of parents (2). Children from low-income families and ethnic minorities have been shown to have poor oral hygiene, infrequent dental visits, less use of preventive dental services and higher untreated carious lesions $(1,3)$. Similarly, Saied Moallemi et al. (4) found direct relationship between the parents' high educational level and the presence of restoration in their children's deciduous teeth. In addition to socio-economic determinants of health, special attention arose toward the role of health literacy on public

\section{Corresponding author:}

Assistant Professor Dr. Mohammad Mehdi Naghibi Sistani, Oral Health Research Center, Department of Community Oral Health, Faculty of Dentistry, Babol University of Medical Sciences, Babol, Iran.

Tel: +98.9153012975, Fax: +98.1132291093, Email: m.naghibi@mubabol.ac.ir

Received: July 31, 2016, Accepted: October 08, 2016, Published: December 2016

iThenticate screening: October 07, 2016, English editing: November 04, 2016, Quality control: December 02,2016

(C) 2016 The Authors. This is an open access article under the terms of the Creative Commons Attribution-NonCommercialNoDerivs License, which permits use and distribution in any medium, provided the original work is properly cited, the use is non-commercial and no modifications or adaptations are made. 
health (5). Adapted from health literacy background, Oral Health literacy is "the degree to which individuals have the capacity to obtain, process, and understand basic health information and services, needed to make appropriate health decisions" (6). It has recently been considered as an important determinant of oral health (7). Respectively, evidence has linked caregiver health literacy to their children's oral health status. Lower caregivers' oral health literacy was associated with a child's high caries risk related behavior such as use of night time bottle and absence of daily teeth or gums brushing (8). High caregiver oral health literacy was also negatively correlated with their children's DMFT index and visible plaque index (9). While, studies on oral health literacy are gradually becoming worldwide, data on this topic is rare in Iran. Study of health literacy in five provinces in Iran revealed that two-thirds of adults had inadequate levels of health literacy (10). Sistani et al. (11) also found a low average of oral health literacy levels among Iranian adults, specifically men, the less educated and those receiving oral health information from few sources. However, no studies have been conducted about the effect of parents' oral health literacy on children's oral health in Iran. Therefore, the aim of this study was to evaluate the effect of parents' oral health literacy on children's dental health.

\section{Material and Methods}

A total of 384 children aged 21 months to 84 months who attended the dental clinic of Babol University of Medical Sciences, Babol, Iran, between September 2015 and February 2016 were examined. The examiner was a final year undergraduate dental student who, before initiating the project, participated in some training sessions such as DMFT index workshop, and the questionnaire protocols which had all been explained by the main researcher at the dental faculty. The response rate was near one hundred percent. Patients who attended the pediatric clinic faculty of dentistry were selected, using non randomized simple method. Because preschool children's oral health is mostly controlled by parents (12), we selected this age group in order to show parents' long-term supervision of children's oral health. Clinical data measured only for primary dentition as $d$ (dental caries of deciduous teeth), $\mathrm{m}$ (missing deciduous teeth due to dental caries), and $\mathrm{f}$ (filling of deciduous teeth due to dental caries), using standard probe of "WHO" at the dental clinic. During examination children's parents completed the "Oral Health Literacy-Adults Questionnaire" (OHL-AQ), which showed to be valid and reliable to measure oral health literacy skill of adults (13). It contains 17 items in four sections: reading comprehension to assess reading literacy and oral health knowledge; numeracy to assess calculating literacy in a dental prescription, and a mouth-rinse instruction; listening to evaluate effectiveness of communication skills; and decision-making which shows applied literacy skills related to common oral health problems and items extracted from medical history form. The correct answers were scored 1 and those wrong or unanswered, 0 . The total score for the questionnaire ranged from 0 to 17 . For analysis, we reclassified the OHL-AQ scores into two levels: inadequate, 0-11 (combining the categories of "inadequate" and "marginal"); and adequate, 12-17 (13). Socio-demographic data was acquired comprising children's age and gender, parents' educational level, parents" job, and family residential region. Age was categorized as: 21-36 months, and 37-84 months. Parents' educational level was measured by linking both parents' years of education and classified as: Low (both parents under 12 years of education), medium (one parent under 12 years of education), or high (both parents 12 or more years of education). Parents' job was categorized as: both parents' employed, one parent employed, and both parents' unemployed. While both rural and urban residents attended the dental faculty clinic, family residential region was categorized as: rural, or urban. In addition to descriptive statistics, the statistical significance of children's dental caries, missing, and dental fillings mean differences between subgroups was compared by one-way analysis of variance (ANOVA), and an independent-samples t-test. Two multiple linear regression model served to test which variables associated with children's dental caries and dental fillings, controlling for background characteristics and oral health literacy level. The significance was set at $<0.05$. All data were analyzed by IBMC SPSS $\odot$ Statistics version 22 (IBM@ Corp., Armonk, NY, USA). The ethics committee of the Babol University of Medical Sciences approved the study. Before being invited to take part, participants were informed of the scientific goal of this research, participation being voluntary and their right to withdraw at any time.

\section{Results}

In all, 384 children (47\% girls) aged 21-84 months participated, while $85 \%$ were $37-84$ months old. The mean age was 55.1 (SD: 13.7). Of the examined children, mean dental caries was 6.5 , mean missing was 0.4 , mean filling was 1.2 , and mean $\mathrm{dmft}$ index was 8.2, respectively. Unemployed parents $(\mathrm{p}<0.001)$, Parents with low level of education $(\mathrm{p}=0.001)$, and those who were living in rural regions $(\mathrm{p}=0.001)$, had children with higher dental caries (Table 1$)$. Girls $(\mathrm{p}=0.02)$ and younger ones $(\mathrm{p}<0.001)$ showed less dental caries, while children who were living in urban regions had more dental fillings $(\mathrm{p}=0.002)$ (Table1). The mean OHL of parents was 11.5, ranged 1 to17. Parents with inadequate OHL had children with more dental caries $(\mathrm{p}=0.005)$, while parents with adequate OHL had children with more dental fillings $(\mathrm{p}=0.001)$ (Table1). With regards to the multiple linear regression results, 
unemployment of both parents was significantly associated with increased children's dental caries $(\mathrm{p}<0.001,95 \%$ CI: 5.64 to 13.45 ) (Table 2), living in urban regions ( $\mathrm{p}=0.01,95 \%$ CI: 0.11 to 1.12 ), and parents with adequate OHL ( $p=0.02,95 \%$ CI: 0.08 to 1.05 ) were significantly associated with more children's dental fillings (Table 3 ).

Table 1. Mean dental caries, missing, and filling according to children's socio-demographic backgrounds and their parents' characteristics $(\mathrm{n}=384)$

\begin{tabular}{|c|c|c|c|c|c|c|c|c|}
\hline \multirow{2}{*}{\multicolumn{2}{|c|}{ Variables }} & \multirow{3}{*}{58} & \multicolumn{2}{|l|}{$\mathrm{d}$} & \multicolumn{2}{|l|}{$\mathrm{m}$} & \multicolumn{2}{|l|}{1} \\
\hline & & & & & & & & \\
\hline Chil & $21-36$ & & \begin{tabular}{|l|}
$\begin{array}{l}\text { Mean } \\
(\mathrm{SD})\end{array}$ \\
$4.5(3.6)$ \\
\end{tabular} & $\begin{array}{l}\text { p-value } \\
-\end{array}$ & $\begin{array}{l}\begin{array}{l}\text { Mean } \\
(\mathrm{SD})\end{array} \\
0.6(1.5)\end{array}$ & $\begin{array}{l}\text { p- } \\
\text { value } \\
-\end{array}$ & $\begin{array}{l}\begin{array}{l}\text { Mean } \\
(\mathrm{SD})\end{array} \\
1.2(2.7)\end{array}$ & $\begin{array}{l}\mathrm{p}- \\
\text { value } \\
-\end{array}$ \\
\hline (months) & $37-84$ & 326 & $6.9(4.4)$ & $<0.001^{*}$ & $0.4(1)$ & $0.2 *$ & $1.2(2.1)$ & $0.9^{*}$ \\
\hline \multirow[t]{2}{*}{ Children's gender } & Boy & 202 & $7(4.6)$ & - & $0.4(1.1)$ & - & $1.1(2.2)$ & \\
\hline & Girl & 182 & $6(4.1)$ & $0.02 *$ & $0.4(1.1)$ & $0.7^{*}$ & $1.2(2.2)$ & $0.6^{*}$ \\
\hline \multirow{2}{*}{ Residential region } & Rural & 123 & $7.6(4.8)$ & - & $0.5(1.2)$ & - & 0.7 (1.7) & - \\
\hline & Urban & 261 & $6(4.1)$ & $0.001 *$ & $0.4(1)$ & $0.4 *$ & $1.4(2.3)$ & $0.002 *$ \\
\hline \multirow[t]{3}{*}{ Parents' job } & $\begin{array}{l}\text { Both } \\
\text { unemployed }\end{array}$ & 5 & $\begin{array}{l}16.2 \\
(3.4)\end{array}$ & - & $0.2(0.8)$ & - & $0(0)$ & - \\
\hline & One employed & 294 & $6.7(4.4)$ & - & $0.591 .2)$ & - & $1.1(2)$ & - \\
\hline & Both employed & 85 & $5.2(3.6)$ & $<0.001 * *$ & $0(0)$ & $0.1^{* *}$ & $1.3(2.7)$ & $0.4^{* *}$ \\
\hline \multirow[t]{3}{*}{ Parents' education } & Low & 36 & $9(4.3)$ & - & $0.3(0.7)$ & - & $1(1.8)$ & - \\
\hline & Medium & 254 & $6.5(4.3)$ & - & $0.4(1.2)$ & - & $1.1(2.2)$ & - \\
\hline & High & 94 & $5.6(4.3)$ & $0.001 * *$ & $0.4(1)$ & $0.7^{* *}$ & $1.3(2.1)$ & $0.7^{* *}$ \\
\hline \multirow{2}{*}{$\begin{array}{l}\text { Parents' } \\
\text { Oral Health Literacy }\end{array}$} & Inadequate & 181 & $7.2(4.5)$ & - & $0.4(1)$ & - & $0.9(1.7)$ & - \\
\hline & Adequate & 203 & $5.9(2.2)$ & $0.005^{*}$ & $0.5(1.2)$ & $0.4^{*}$ & $1.4(2.5)$ & $0.01 *$ \\
\hline
\end{tabular}

* Independent sample t-test; **One way ANOVA

Table 2. Factors related to children's dental caries controlling for residential area and parents' characteristics by multiple linear regression $(\mathrm{n}=384)$

\begin{tabular}{|l|l|l|l|l|}
\hline Variables & Regression coefficient & $95 \%$ CI & p-value \\
\hline \multirow{3}{*}{ Parents' job } & Both unemployed & 9.54 & 5.64 to 13.45 & $<0.001$ \\
\cline { 2 - 6 } & One employed & 0.83 & -0.35 to 2.02 & 0.16 \\
\cline { 2 - 6 } & Both employed & Referent & - & - \\
\hline \multirow{3}{*}{ Parents' education } & Low education & 1.60 & -0.24 to 3.46 & 0.09 \\
\cline { 2 - 6 } & medium education & 0.003 & -1.17 to 1.17 & 0.99 \\
\cline { 2 - 6 } & high education & Referent & - & - \\
\hline Residential region & Urban & -0.86 & -1.83 to 0.09 & 0.07 \\
\cline { 2 - 6 } & Rural & referent & - & - \\
\hline Parents' Oral health literacy & Adequate & -0.70 & -1.63 to 0.22 & 0.13 \\
\cline { 2 - 6 } & Inadequate & referent & - & - \\
\hline
\end{tabular}

Table 3. Factors related to children's dental fillings controlling for residential area and parents' characteristics by multiple linear regression $(\mathrm{n}=384)$

\begin{tabular}{|l|l|l|l|l|}
\hline Variables & Regression coefficient & $95 \%$ CI & p-value \\
\hline \multirow{3}{*}{ Parents' job } & Both unemployed & -0.92 & -2.97 to 1.12 & 0.37 \\
\cline { 2 - 5 } & One employed & 0.10 & -0.52 to 0.72 & 0.74 \\
\cline { 2 - 6 } & Both employed & Referent & - & - \\
\hline \multirow{3}{*}{ Parents' education } & Low education & 0.29 & -0.68 to 1.26 & 0.55 \\
\cline { 2 - 6 } & Medium education & 0.16 & -0.45 to 0.77 & 0.60 \\
\cline { 2 - 6 } & High education & Referent & - & - \\
\hline \multirow{2}{*}{ Residential region } & Urban & 0.61 & 0.11 to 1.12 & 0.01 \\
\cline { 2 - 6 } & Rural & Referent & - & - \\
\hline \multirow{2}{*}{ Parents' Oral health literacy } & Adequate & 0.57 & 0.08 to 1.05 & 0.02 \\
\cline { 2 - 6 } & Inadequate & Referent & - & - \\
\hline
\end{tabular}




\section{Discussion}

The present study results revealed that inadequate parents' OHL was associated with high children's dental caries and less dental fillings. The regression model, however, confirmed significant relation of lower parents' OHL and less children's dental fillings controlling for family's socio-economic determinants. Previous studies also linked inadequate caregiver's OHL to high children's dental treatment needs (3), high children's dmft index (9), and adverse early childhood oral health outcome (8). In addition, we found that, parents' low educational level, parents' unemployment, and living in rural regions was associated with higher children's dental caries status. In regression model however, parents' unemployment remained as a significant related factor to higher children's dental caries, indicating a notable role of economic status in children's oral health compared to other socio-demographic determinants. Similarly, Bridge et al. (9) found that low parents' educational attainment and low family income were associated with high children's dental caries status. Considering our study, children's oral health from their living areas revealed high dental caries and low dental fillings of rural children compared to those living in urban areas. Availability of unhealthy and sugary foods and beverages in villages similar to major cities, limited dental health services in rural regions (14), and lack of oral health prevention knowledge may contribute to poor children's oral health in rural communities. While reducing dental health services disparities in rural areas faces a multitude of challenges, regarding the present study results, improving OHL of deprived individuals seems to carry a lot of potential to promote children's oral health, specifically in countries with a developing oral health care system, such as Iran. Providing interventions in settings such as health care centers where pregnant women and children under six years referred regularly, would be beneficial for improving caregivers, specifically mothers' OHL skills. Adequate mothers' skills in relation to their child's oral health, leads to improved health practices in mother-child pairs (2). Van et al. (8) also concluded that interventions to enhance caregivers' oral health literacy would be a successful strategy to improve young children's oral health. The results of the present study showed an increase in the rate of dental caries with children's aging, which might be attributed to prolonged exposure to caries risk factors and parents' control over their children's nutrition and the child's hygienic behavior decreasing as they grow up (12). The present study was limited to cross-sectional design and all the subjects were examined from patients referring to the Specialty Clinic of Babol Faculty of Dentistry. Consequently, the results may not be possible to be extended to whole populations and should be interpreted cautiously.

\section{Conclusions}

Our study shows that a low level of parents' OHL and living in a rural area was significantly associated with less children's dental fillings. We also found that parents' unemployment was significantly related to higher children's dental caries. Considering that changing families' residential and economic situation seems to be impossible, improving parents' OHL may be a useful alternative to promote children's dental health status. Since, studies on health literacy, especially on oral health literacy are rare, it is suggested that further studies be carried out on this topic in different parts of the country, with large sample sizes and on different age groups.

\section{Acknowledgments:}

This research was supported by a Babol University of Medical Sciences \& Health Services grant (MUBABOL.REC.1395.103). The authors wish to thank Dr. M. Hamze and Dr. S. Gharekhani for their contribution in data collection.

\section{Conflict of Interest:}

There is no conflict of interest to be declared.

\section{Authors' contributions:}

All authors contributed to this project and article equally. All authors read and approved the final manuscript.

\section{References:}

1) Mouradian WE, Wehr E, Crall JJ. Disparities in children's oral health and access to dental care. JAMA. 2000; 284(20): 2625-31. doi: 10.1001/jama.284.20.2625. PMID: 11086371.

2) Mohebbi SZ, Virtanen JI, Murtomaa H, Vahid-Golpayegani M, Vehkalahti MM. Mothers as facilitators of oral hygiene in early childhood. Int J Paediatr Dent. 2008; 18(1): 48-55. doi: 10.1111/j.1365263X.2007.00861.x. PMID: 18086026.

3) Miller E, Lee JY, DeWalt DA, Vann WF Jr. Impact of caregiver literacy on children's oral health outcomes. Pediatrics. 2010; 126(1): 107-14. doi: 10.1542/peds.2009-2887. PMID: 20547644, PMCID: PMC2896459. 
4) Saied-Moallemi Z, Virtanen JL, Tehranchi A, Murtomaa H. Disparities in oral health of children in Tehran, Iran. Eur Arch Paediatr Dent. 2006; 7(4): 262-4. doi: 10.1007/BF03262563. PMID: 17164073.

5) Divaris K, Lee JY, Baker AD, Vann WF Jr. The relationship of oral health literacy with oral health-related quality of life in a multi-racial sample of low-income female caregivers. Health Qual Life Outcomes. 2011; 9: 108. doi: 10.1186/1477-7525-9-108. PMID: 22132898, PMCID: PMC3248838.

6) US Department of Health and Human Services, Healthy people 2010: oral health toolkit. Washington, DC: U.S. Government Printing Office. 2000.

7) Naghibi Sistani MM, Yazdani R, Virtanen J, Pakdaman A, Murtomaa H. Determinants of oral health: does oral health literacy matter? ISRN Dent. 2013; 2013: 249591. doi: 10.1155/2013/249591. PMID: 23577262, PMCID: PMC3610341.

8) Vann WF Jr, Lee JY, Baker D, Divaris K. Oral health literacy among female caregivers: impact on oral health outcomes in early childhood. J Dent Res. 2010; 89(12): 1395-400. doi: 10.1177/0022034510379601. PMID: 20924067, PMCID: PMC3123718.

9) Bridges SM, Parthasarathy DS, Wong HM, Yiu CK, Au TK, McGrath CP. The relationship between caregiver functional oral health literacy and child oral health status. Patient Educ Couns. 2014; 94(3): 411 6. doi: 10.1016/j.pec.2013.10.018. PMID: 24308901.

10) Tehrani Banihashemi SA, Amirkhani MA, Haghdoost AA, Alavian SM, Asgharifard H, Baradaran H, et al. Health literacy and the Influencing Factors: A study in five provinces of Iran. Int J Prev Med. 2012; 3(2): 116-21. PMID: 22347608.

11) Sistani MM, Yazdani R, Virtanen J, Pakdaman A, Murtomaa H. Oral health literacy and information sources among adults in Tehran, Iran. Community Dent Health. 2013; 30(3): 178-82. PMID: 24151793.

12) Casamassimo PS, Fields HW, McTigue DJ, Nowak A. Pediatric Dentistry Infancy Through Adolessence. Elsevier Saunders. 2013; 160-66.

13) Naghibi Sistani MM, Montazeri A, Yazdani R, Murtomaa H. New oral health literacy instrument for public health: development and pilot testing. J Investig Clin Dent. 2014; 5(4): 313-21. doi: 10.1111/jicd.12042. PMID: 23559571.

14) Kiadaliri AA, Hosseinpour R, Haghparast-Bidgoli H, Gerdtham UG. Pure and social disparities in distribution of dentists: a cross-sectional province-based study in Iran. Int J Environ Res Public Health. 2013; 10(5): 1882-94. doi: 10.3390/ijerph10051882. PMID: 23648443, PMCID: PMC3709354. 